\title{
VALIDATING AN INTEGER NON-LINEAR PROGRAM OPTIMIZATION MODEL OF A WIRELESS SENSOR NETWORK USING AGENT-BASED SIMULATION
}

\author{
Mumtaz Karatas \\ Department of Industrial Engineering \\ Turkish Naval Academy \\ Tuzla, Istanbul, 34942, TURKEY
}

\author{
Bhakti Stephan Onggo \\ Department of Management Science \\ Lancaster University Management School \\ Lancaster University \\ Lancaster, LA1 4YX, UNITED KINGDOM
}

\begin{abstract}
Deploying wireless sensor networks (WSN) along a barrier line to provide surveillance against illegal intruders is a fundamental sensor-allocation problem. To maximize the detection probability of intruders with a limited number of sensors, we propose an integer non-linear program optimization model which considers multiple types of sensors and targets, probabilistic detection functions and sensor-reliability issues. An agent-based simulation (ABS) model is used to validate the analytic results and evaluate the performance of the WSN under more realistic conditions, such as intruders moving along random paths. Our experiment shows that the results from the optimization model are consistent with the results from the ABS model. This increases our confidence in the ABS model and allows us to conduct a further experiment using moving intruders, which is more realistic, but it is challenging to find an analytic solution. This experiment shows the complementary benefits of using optimization and ABS models.
\end{abstract}

\section{INTRODUCTION}

A Wireless Sensor Network (WSN) consists of spatially distributed autonomous sensors that are generally used for monitoring or surveillance purposes. Given the wide range of WSN characteristics and capabilities, they are actively used in many applications, such as battlefield surveillance, intruder detection along international borders, public health, healthcare, biological detection, home appliances, smart spaces and inventory tracking (Cardei and Wu 2006). As a consequence of these diverse application fields the requirements and operational constraints imposed on WSN deployment vary. Hence, deployment issues such as coverage, topology control, connectivity maintenance, routing, security and data management are challenging ( $\mathrm{Vu} 2009$ ). Among these issues, the coverage problem is one of the most common issues when WSN is used for surveillance and the detection of intruders.

WSN coverage problems can be categorized into three groups: area coverage, point coverage and barrier coverage. In an area-coverage problem, the goal is generally to achieve the maximum coverage level or guarantee a certain level of coverage in the area of interest. Some important applications include habitat monitoring (Szewczyk et al. 2004), volcano monitoring networks (Werner-Allen et al. 2006), forest-fire applications (Yu, Wang, and Meng 2005), and a study of birds' behaviour (Kumagai 2004). In a point (target) coverage scenario, the search space consists of a discrete set of locations and the objective is to create a certain level of surveillance on or around such locations. The coverage status or probability of a target can easily be determined by comparing the sensor detection range and the distance between a target and a sensor. In this type of problem, a decision-maker is mainly interested in the detection of intruders around critical infrastructure facilities and geographic delimiters, such as 'high value' facilities and chemical plants. In a barrier-coverage problem, the objective is to prevent an intruder (or mobile 


\section{Karatas and Onggo}

target) penetrating a belt region or a one-dimensional line segment that separates one region from another. Barrier coverage has a number of advantages over area coverage. The first is that fewer sensors are needed in comparison to full area coverage (Kumar, Lai, and Arora 2005). The second advantage is that the sleep-wakeup schedule problem in barrier coverage is polynomial-time solvable, whereas it is NPhard for area coverage (Kumar et al. 2007; Chen, Kumar, and Lai 2010).

Typically, a WSN comprises hundreds of individual sensors that must be deployed carefully in order to achieve a certain objective. However, due to operational or tactical factors (such as a tight deployment budget, limited accessibility to the field or limited operation time), sensors are often deployed randomly or in regular patterns without considering optimality (He et al. 2012). One major drawback of such a deployment strategy is the possibility of sensing holes or weak surveillance where an intruder is able to penetrate the barrier. Furthermore, sensors are imperfect. Several factors, such as heat, defective components, malicious activity, environmental hazards, extended use or lack of power, may cause sensors to fail (Chen, Lai, and Xuan 2008). These reliability factors make the sensing-holes problem more severe. It is also known that a given type of sensor has different detection accuracy for different types of targets (i.e. sensitivity and specificity). This issue makes finding the optimal allocation of different types of sensors in a WSN barrier against different types of intruders more difficult. The difficulty is further increased when a probabilistic detection function is used (i.e. detection probability is a function of the distance between target and sensor). To our knowledge, there is no existing study that deals with the optimization and performance measurement of a WSN barrier that takes into account multiple unreliable sensor types with probabilistic detection functions and different types of targets.

Motivated by the lack of research in this area, we are interested in studying the problem of determining and measuring the performance of optimal WSN layouts for barrier-coverage purposes. Specifically, in this paper, we consider a set of sensors (of multiple types) and a number of candidate sensor locations along a barrier line. Each sensor type has a unique probabilistic detection function for each target type. We further assume that the sensors are not perfect and may fail during a search operation. The objective of a decision-maker is to maximize the total expected-target (intruder) detection probability by choosing $m$ sensor locations from among $n$ candidates. In order to solve the problem, we develop an Integer Non-Linear Program (INLP). The analytic results are compared with an agent-based simulation (ABS) model that is developed using the same assumptions, which provides cross-validation of the two models. Having validated the ABS model, we evaluate the performance of WSN by making more realistic assumptions (i.e. moving targets) using the ABS model. This study shows the benefits of adopting a multi-methodology approach (Mustafee and Bischoff 2013).

This paper is organized as follows. In Section 2, we review previous work on the barrier-coverage problem in WSNs. The details of our optimization and simulation models are presented in Section 3. Section 4 discusses the validation of the models and the results of our experiments. Finally, we present the conclusion of our work in Section 5.

\section{LITERATURE REVIEW}

Gage (1992) was the first to introduce the concept of barrier coverage in a study that aimed to detect intruders crossing a barrier that divides a network into two regions. A more recent study extended the work to the WSN barrier-coverage problem with a two-dimensional plane and a two-dimensional belt region (Liu and Towsley 2004). Since then, there has been various work that addresses how to maximize coverage (e.g. Kumar, Lai, and Arora 2005; Liu et al. 2008; Bereg and Kirkpatrick 2009). Coverage is not the only factor when considering the placement of WSNs, they need to be energy efficient. This is provided by a smart sleep-wakeup schedule. Sleep-wakeup is used to calculate a subset of sensors that will be put to sleep while others remain active at any given time. Kumar et al. (2007) developed algorithms that determine the optimal sleep-wakeup schedule of WSNs that can be completed in polynomial-time. They extended their work to find solutions for both homogeneous and heterogeneous lifetime cases (Kumar et al. 2010). The reliability of WSNs is also an important factor. In their study, 


\section{Karatas and Onggo}

Chen, Lai, and Xuan (2008) discuss the reliability and quality of barrier coverage using WSNs. They proposed a technique to determine weak zones that need to be repaired and discuss how to repair a weak region. Budget constraints are also an important issue in WSN deployment. He et al. (2012) studied a barrier-coverage problem where the number of available sensors was insufficient. They proposed a periodic monitoring scheduling (PMS) algorithm that improves coverage performance by jointly exploiting sensor mobility and intruder-arrival information. They designed a coordinated sensorpatrolling (CSP) algorithm that further improves performance significantly. Ma et al. (2012) considered the Minimum Camera Barrier Coverage Problem (MCBCP) for randomly deployed WSNs in a target area. They proposed an optimal algorithm for the minimum-weight path problem and found a feasible solution to it.

There are a number of articles that discuss the performance of randomly deployed sensors. For example, Saipulla et al. (2008) studied the barrier-coverage performance of airdropped WSNs. They assume that sensor positions are normally distributed along a barrier line. They analyzed the impact of variance on network performance. In another study, Saipulla et al. (2009) analyzed WSN barrier coverage where sensors are deployed along a barrier line with normally distributed random offsets. The authors also considered the multiple-barrier deployment scenario and studied the effect of track spacing between barrier lines on the performance of the barrier. Ssu et al. (2009) also considered randomly deployed WSN barriers and aimed to generate $k$-barrier coverage with a directional sensing model. They show that it is possible to achieve $k$-barrier coverage with fewer sensors than the omni-directional sensing model.

\section{METHODOLOGY}

\subsection{Detection Model}

In real life, intruders are highly likely to follow the shortest path across a barrier line or belt region (Chen, Kumar, and Lai 2010). Thus, it is reasonable to assume that intruders move in straight lines perpendicular to a barrier line. This assumption enables us to compute the cumulative detection probability (CDP) $p$ of an intruder by a sensor along its continuous track. Each sensor type, in a given environment and for a given target type, will have a detection-probability curve that describes its performance as a function of $d$, which is the closest point of approach (CPA) between a sensor and the intruder's path. These curves can have many shapes. We consider a sensor model in which the probability of detection is an arbitrary nonincreasing function. Numerous empirical formulas exist in the literature for detection probability, e.g. polynomial, exponential and cubic attenuation models. In this paper, we adopt the exponential probabilistic detection model. This model is conservative in terms of estimating sensing capability and has also been used several times to model the probabilistic coverage of WSNs (e.g. Li, Chen, and Lai 2012; Ahmed, Kanhere, and Jha 2005; Zou and Chakrabarty 2004; Zou and Chakrabarty 2005). The equation used in exponential detection function is $p=\exp (-\alpha d)$ where $\alpha$ denotes the sensor capability by describing how fast the sensing capability decays with distance $d$.

Throughout this work, we assume that the detection of a target by a particular sensor is independent of its detection by other sensors. Under this assumption, the non-detection probability of a target can be computed as the product of the individual detection probabilities of all sensors. Therefore, for a given set of sensors $m \in M$, the CDP of a target trying to penetrate the barrier is given by (1):

$$
P=1-\prod_{m \in M}\left(1-p_{m}\right)
$$

where $p_{m}$ denotes the individual detection probability by sensor $m$.

Figure 1 shows an example of a CDP function from two sensors. We observe that the detection probability at any point along the barrier segment is enhanced by contributions from the two neighbouring sensors. Additionally, the CDP at any point along the barrier segment can be further increased by 


\section{Karatas and Onggo}

decreasing the distance between the two neighbouring sensors to the detriment of the CDP at the two ends of the barrier segment.

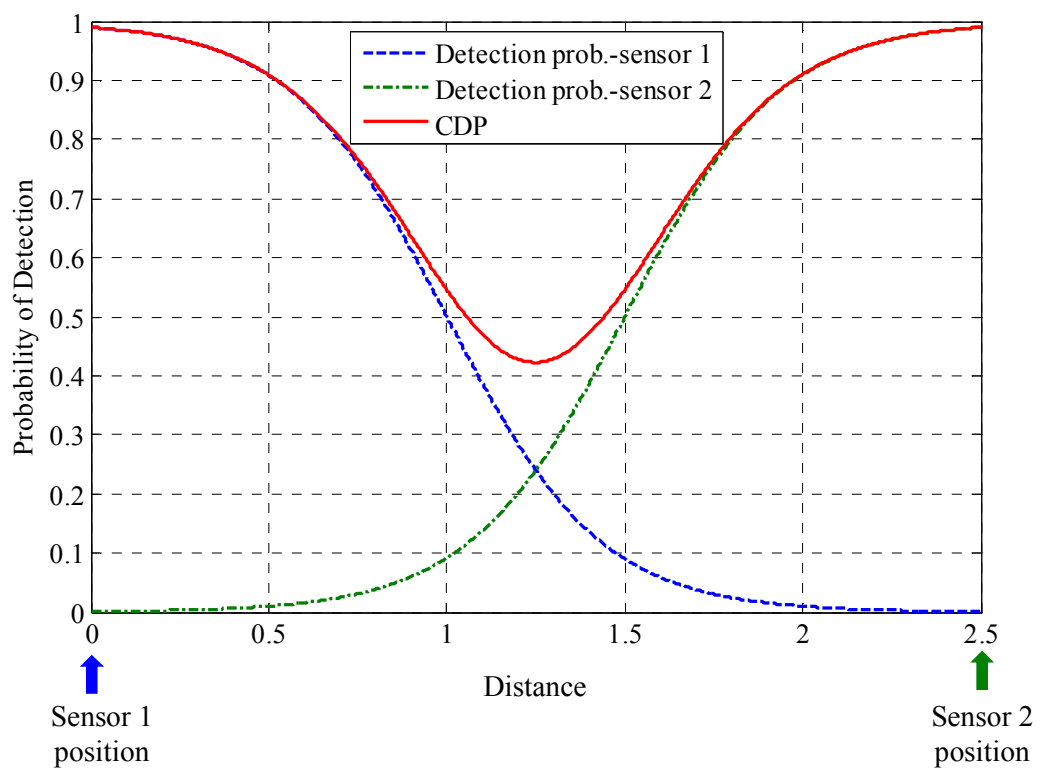

Figure 1: Exemplary CDP of a target by two sensors along a barrier segment.

\subsection{Optimization Model}

In our model, we consider a set of sensors and a set of targets in a two-dimensional Euclidean plane. We have a set of available sensors and would like to place them in such a way as to achieve good detection probability of the targets. We denote the set of sensor types as $I$, indexed by $i \in I$, the set of target types $J$, indexed by $j \in J$, and the set of candidate sensor locations as $S$, indexed by $s \in S$. In order to model the possible paths of targets, we discretize the barrier-line segment into small intervals and denote the set of target paths as $T$, indexed by $t \in T$. Each target type $j$ is associated with a weight $v_{j}$ that reflects its value.

We further introduce the concept of reliability for each sensor type and associate each sensor type $i$ with a pre-determined reliability coefficient $\beta_{i}$ such that $0 \leq \beta_{i} \leq 1, \forall i$. Additionally, we assume that each target type $j$ has a unique detection probability function with respect to a sensor type $i$. We model the differences in detection capabilities by assigning a certain $\alpha_{i j}$ value to each pair of sensor $i$ and target $j$. Thus, we calculate $p_{i j s t}$, the CDP of a target type $j$ following path $t$ with sensor type $i$ located at position $s$ as in equation (2):

$$
p_{i j s t}=\exp \left(-\alpha_{i j} d_{s t}\right)
$$

Figure 2 is an exemplary barrier-line segment of length $L$. The squares represent candidate sensor locations and each dashed arrow pointing down represents a discretized possible target path. The example allocation plan in the figure shows the locations of two types of sensors along with their approximate CDFs for the two target types.

Given a finite set of candidate sensor locations, we now turn our attention to developing an optimization model to select the best locations for our sensors. The model is formulated with sets and indices, parameters, variables, an objective function and constraints, as explained below. 


\section{Karatas and Onggo}

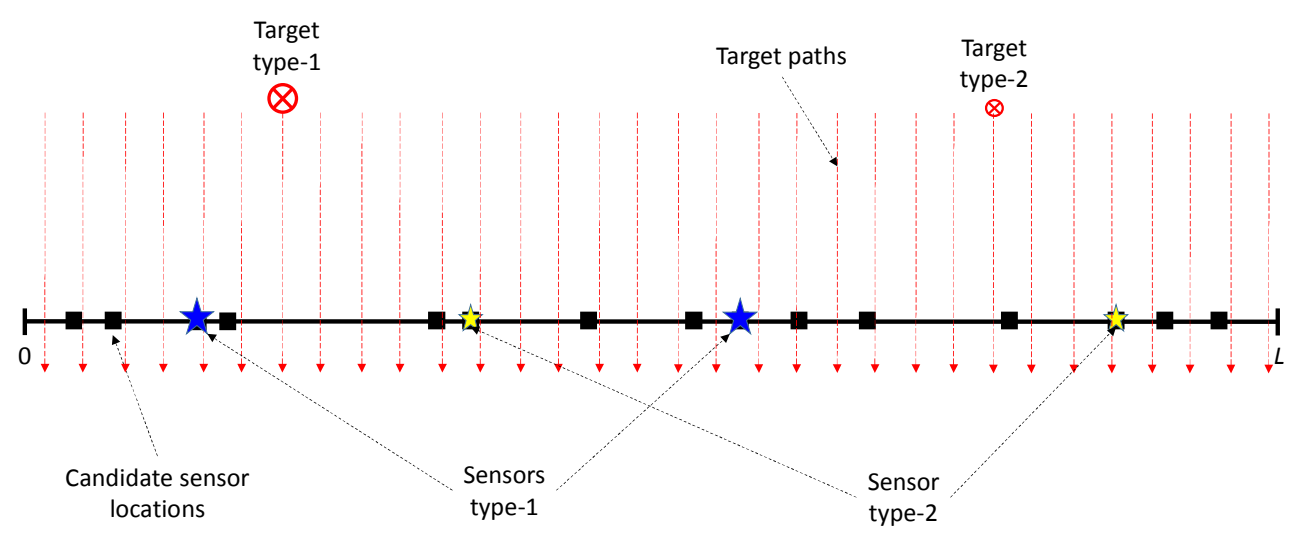

Figure 2: An exemplary WSN barrier-problem set-up. Candidate sensor locations and actual sensor locations are represented by squares and stars, respectively.

Sets and Indices:

$s \in S:$ set of candidate sensor locations

$t \in T:$ set of target paths

$i \in I$ : set of sensor types

$j \in J$ : set of target types

Parameters:

$K_{i} \quad=$ number of available sensors of type $i$

$v_{j} \quad=$ weight of target type $j$

$d_{s t}=$ lateral distance between sensor position $s$ and target path $t$

$p_{i j s t}=$ cumulative detection probability of target $t$ of type $j$ by sensor of type $i$ located at $s$

$\lambda_{j} \quad=$ probability that a target is of type $j$

$\beta_{i} \quad=$ reliability of sensor type $i$

Decision Variables:

$x_{i s}= \begin{cases}1, & \text { a type } i \text { sensor is placed at location } s \\ 0, & \text { otherwise }\end{cases}$

Objective function:

$$
\max _{\mathrm{x}} z=\sum_{j \in J}\left\{\lambda_{j} v_{j} \sum_{t \in T}\left[1-\prod_{s \in S} \prod_{i \in I}\left(1-\beta_{i} x_{i s} p_{i j s t}\right)\right]\right\}
$$

Constraints:

$$
\begin{aligned}
& \sum_{s \in S} x_{i s} \leq K_{i}, \quad \forall i \in I \\
& x_{i s} \in\{0,1\}, \forall i \in I, s \in S
\end{aligned}
$$

The objective function (3) seeks to maximize the total weighted-detection probability of targets attempting to penetrate the barrier. The formula assumes that the detection probability of a sensor is independent of the detection probability of other sensors. Note that $p_{i j t}$ is computed by equation (2). Constraint (4) restricts the number of sensors placed. Constraint (5) declares the variable type. 


\subsection{Simulation Model}

We use an ABS model for two purposes: to validate the analytic solution from the INLP model and to test the robustness of the solution using more realistic assumptions (such as moving targets). Before we use the ABS model, we need to validate the model by comparing its results against the INLP optimization model. This is done by applying the same assumptions used in the optimization model to the ABS model (i.e. validation mode). We relax the assumptions when we use the ABS to test the robustness of the results from the optimization model (i.e. experiment mode).

We implement the ABS model using Repast Simphony (North et al. 2013). The user interface is shown in Figure 3. The user interface provides three parameters: the height of the rectangular area on the right (in $\mathrm{km}$ ), the width of the barrier in which sensors are placed along a straight line at the bottom of the rectangular area on the right (in $\mathrm{km}$ ), and the simulation execution mode (i.e. validation mode and various experimental modes).

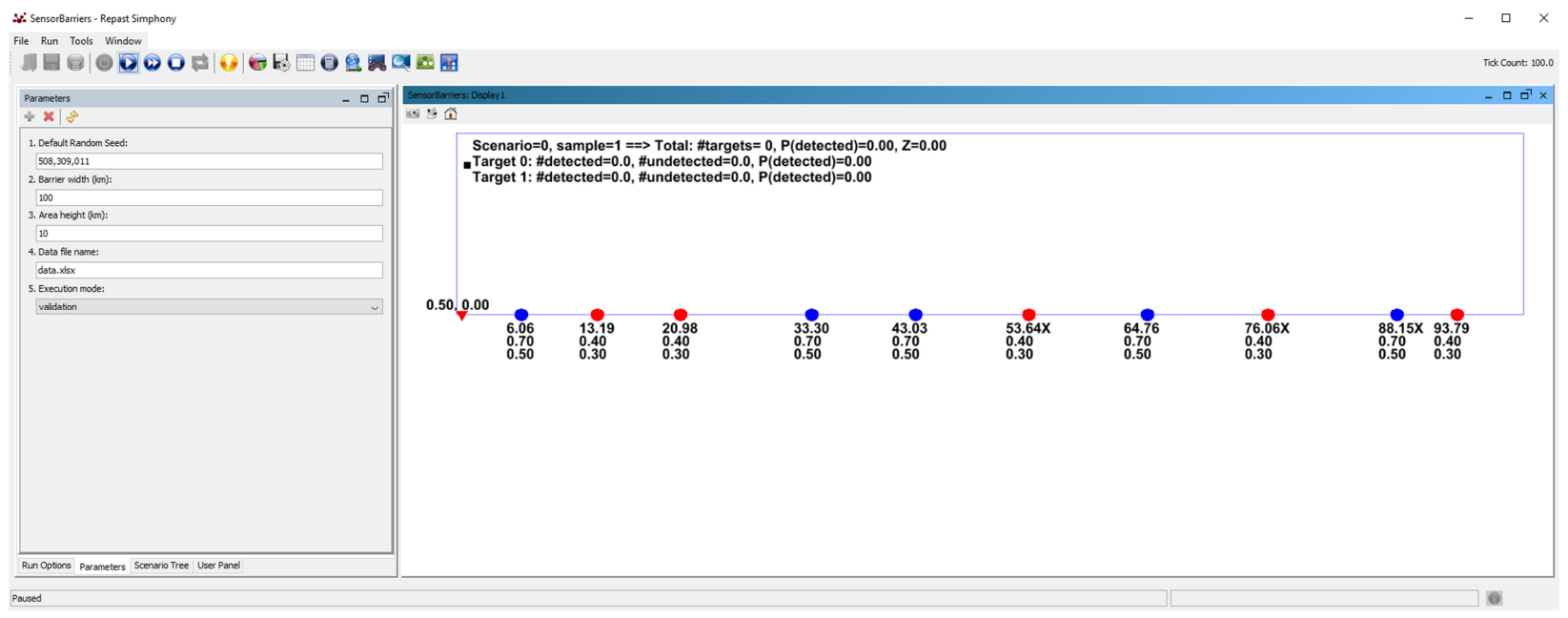

Figure 3: Agent-based sensor-barrier model.

The parameters for the configuration of sensors and target are read from an Excel file to make the configuration task easier for users. Figure 4 shows the structure of the Excel file. The first few columns provide detailed characteristics of the sensors and targets (input to the model). The number of columns depends on the number of sensor and target types (Figure 4 shows two sensor types and two target types). The section on the right of the Excel sheet shows detailed simulation outputs. Each column shows the result from one replication. The number of columns depends on the number of replications. Outputs such as the probability of target types $j\left(\lambda_{j}\right)$ and the probability of sensor $i$ operation $\left(\beta_{i}\right)$ are used in model verification (i.e. making sure all stochastic inputs are sampled correctly) while outputs such as $Z$ are used in model validation (i.e. fit for purpose by making sure that the results are consistent with the INLP results). Each sheet in a workbook represents one validation case. The example given in Figure 4 has five validation cases.

The ABS model is formed by two agent types: sensor and target. Figure 5a shows the behaviour of a sensor. In each time step, a sensor will check if it is operational based on its reliability $\left(\beta_{i}\right)$. If it is operational, it will detect any target with a probability that is calculated using equation (2). If a target is detected then it will be flagged as detected. These steps are repeated until the simulation ends. The behaviour of a target depends on whether we run the simulation using the assumptions used in the optimization model (i.e. validation mode) or use more relaxed assumptions (i.e. various experimental modes). Figure $5 \mathrm{~b}$ shows the behaviour of a target in validation mode. In each time step, we sample a target type $j$ and place it on a path $t$ (we start from a location at the intersection between the leftmost path and the sensor barrier). If the target is detected by any of the sensors, we increase the number of detected 


\section{Karatas and Onggo}

targets. Likewise, if the target is not detected, the number of undetected targets is increased. We repeat this step (sampling target type $j$ and place it at a location on the next path $t$ until all paths are taken). In the example given in Figure 3, we set the number of paths as 100 and the barrier width as $100 \mathrm{~km}$. Hence, we divide the barrier segment into 100 discrete paths. In this case, the first target location is $(0.5,0)$. The next location is $(1.5,0)$ and this step is repeated until the last location $(99.5,0)$. Once we have covered all paths, we can compute the objective function in equation 3 and store the result in row 18 in column $\mathrm{F}$ onwards in an Excel file (see Figure 4). In the next step, we will check if the simulation needs to continue (i.e. do we have enough replications to build a confidence interval for the objective function?).

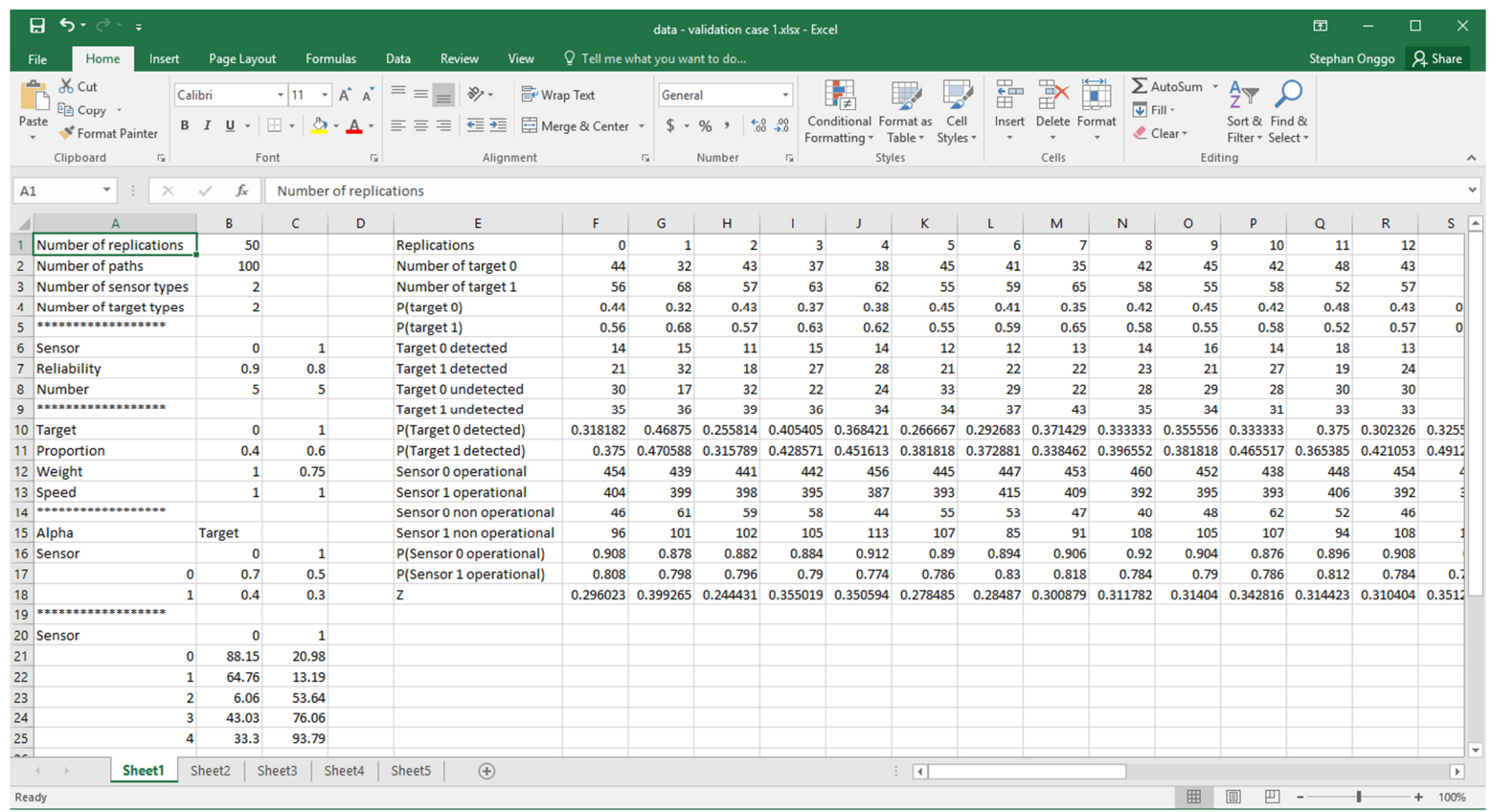

Figure 4: Simulation parameters.

In experiment mode, the behaviour of a target is similar to that in the validation mode, except that the paths are not discretized and the target can be set to move along a path from its original location away from the barrier and to a destination just behind the sensor barrier.

\section{EXPERIMENTAL RESULTS}

In this section we use ABS to validate the INLP model and experiment-barrier performance under more realistic conditions. We use the General Algebraic Modeling System (GAMS) CPLEX 12.2.0.2 to generate problem instances and solve the INLP optimization model. In all experiments, we consider 50 candidate sensor locations on a barrier of length $100 \mathrm{~km}$. and generate $|T|=100$ uniformly distributed target tracks along the barrier line.

\subsection{Validation}

Table 1 shows the parameters used in both INLP and ABS models. The table shows that we have ten cases organized into two groups. The first group (case 1.X) represents a decision to use fewer but more reliable sensors, while the second group (case 2.X) represents the use of more sensors that are less reliable. These cases will be used in the validation and experiments. The validation result is shown in Figure 6 . The figure shows that all optimization results are within the $95 \%$ confidence intervals of the simulation results. In other words, the ABS model can replicate the results from the optimization model well. 
Karatas and Onggo

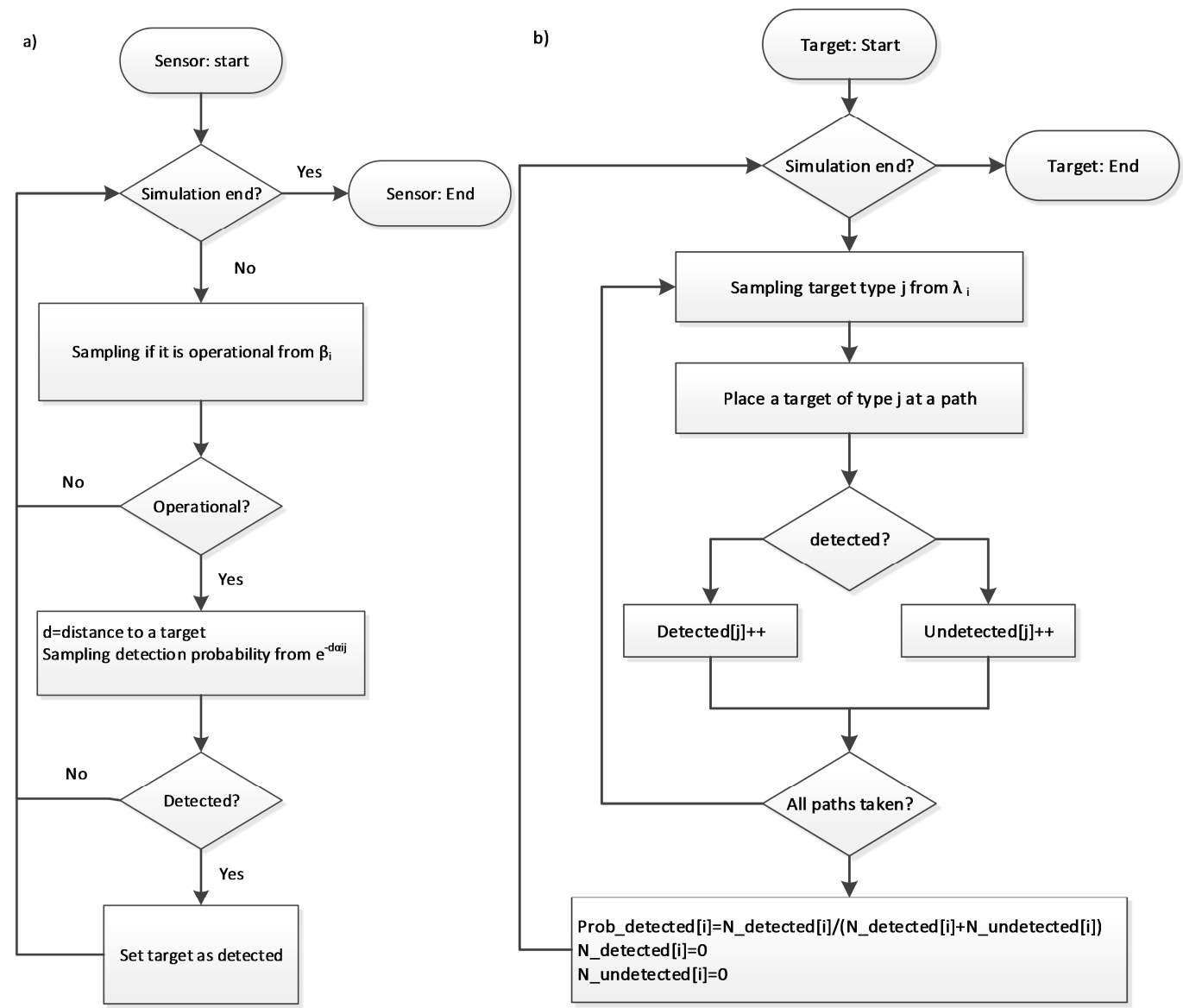

Figure 5: Agent-based simulation - a) sensor's behaviour, b) target's behaviour.

Table 1: Model parameters

\begin{tabular}{|c|c|c|}
\hline \multirow{2}{*}{\multicolumn{2}{|c|}{$\begin{array}{l}\text { All cases } \\
\text { All case } 1\end{array}$}} & $T=\{0.5,1.5,2.5, \ldots, 99.5\}, I=\{0,1\}, J=\{0,1\}, v=\{1,0.75\}, \lambda=\{0.4,0.6\}$ \\
\hline & & $\beta=\{0.9,0.8\}$ \\
\hline \multicolumn{2}{|c|}{ All case 2} & $K=\{10,10\}, \quad \beta=\{0.8,0.6\}$ \\
\hline \multirow{10}{*}{ 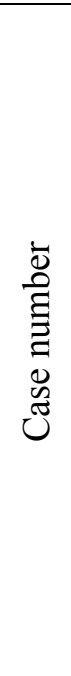 } & 1.1 & $S=\{\{88.15,64.76,6.06,43.03,33.3\}$ \\
\hline & 1.2 & $S=\{\{75.27,95.52,15.62,33.74,40.96\}, \quad\{65.68,49.65,26.7,5.38,87.9\}\}$ \\
\hline & 1.3 & $S=\{\{71.01,78.33,56.99,15.13,21.82\}, \quad\{64.79,6.9,89.6,29.79,42.33\}\}$ \\
\hline & 1.4 & $S=\{\{49.41,60.7,8.55,88.23,96.25\}$ \\
\hline & 1.5 & $S=\{\{10.06,82.99,48.08,54.22,18.49\}$ \\
\hline & 2.1 & $\begin{aligned} S=\{ & \{48.52,89.37,94.92,67.23,22.91,57.22,40.63,85.02,75.79,5.54\}, \\
& \{12.35,43.46,71.63,51.92,62.28,27.67,19.1,81.78,7.39,35.95\}\end{aligned}$ \\
\hline & 2.2 & $\begin{aligned} S=\{ & \{7.37,15.99,9.41,35.25,84.14,79.27,12.9,55.37,39.98,21.97\} \\
& \{86.67,75.9,51.25,59.91,72.3,24.61,66.24,29.49,45.5,89.58\}\}\end{aligned}$ \\
\hline & 2.3 & $\begin{aligned} S=\{ & \{33.9,83.88,45.38,58.02,40.59,11.44,78.95,3.17,51.04,37.55\}, \\
& \{14.32,76.95,58.02,70.9,90.06,27.51,96.95,20.62,6.66,93.15\}\}\end{aligned}$ \\
\hline & 2.4 & $\begin{aligned} S=\{ & \{25.28,2.89,92.76,86.43,50.52,64.46,38.91,22.07,9.8,6.18\}, \\
& \{57.65,29.86,16.85,96.4,88.05,33.24,56.27,72.19,45.59,70.83\}\}\end{aligned}$ \\
\hline & 2.5 & $\begin{aligned} S=\{ & \{73.57,67.85,4.82,85.27,11.71,37.23,32.02,96.89,78.11,94.66\}, \\
& \{82.16,90.65,52.76,57.17,9.25,61.62,43.77,29.33,21.92,17.8\}\}\end{aligned}$ \\
\hline
\end{tabular}




\section{Karatas and Onggo}

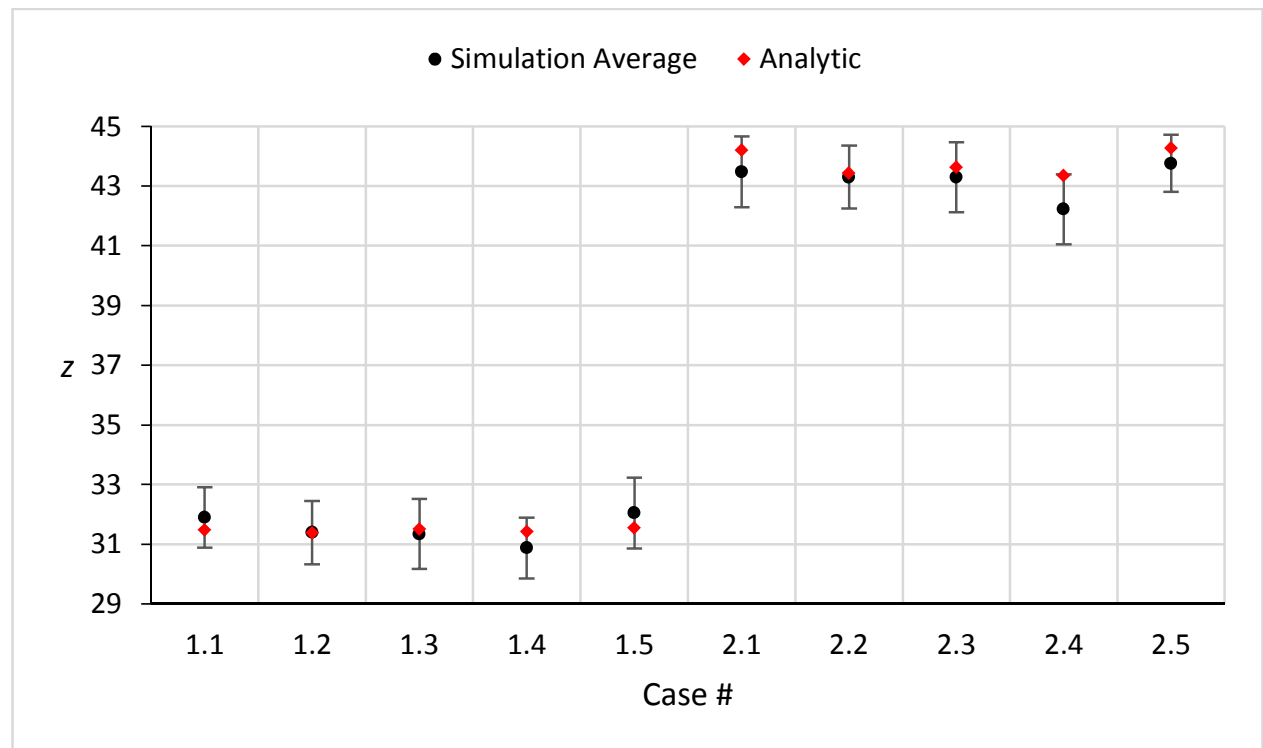

Figure 6: Comparison of analytic model and simulation results (validation) for each test case.

\subsection{Experiment}

In the experiment, we evaluate the robustness of the placement of sensors that is obtained from the optimization model. Figure 7 shows the results for four different cases. The base case refers to the simulation results from Section 4.1 (validation) which replicate the results from the INLP model well. In experiment 1 , the targets are placed at any location along the barrier (i.e. the paths are not discretized). The results are not significantly different from the base case. Hence, the INLP model can provide a good approximation of the condition in which paths are not discretized.

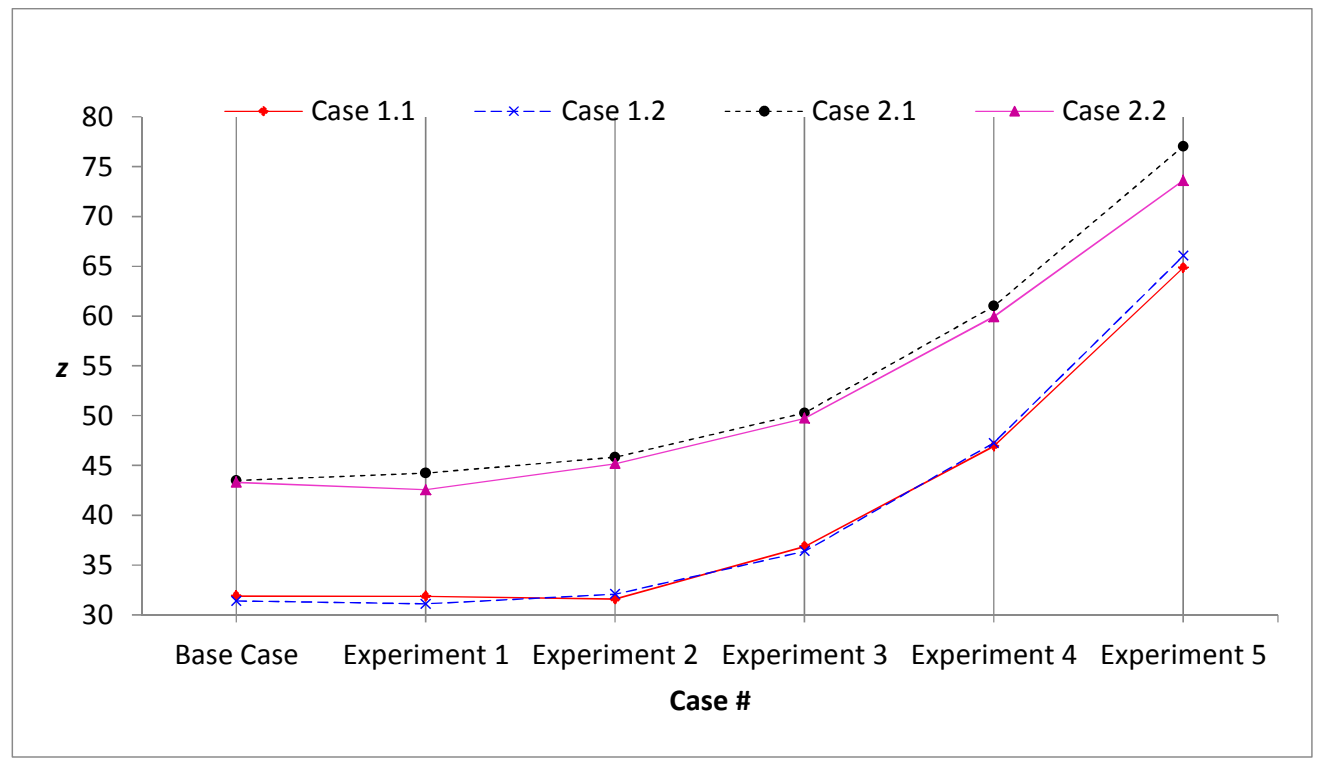

Figure 7: Comparison of analytic model and simulation results (experiment) for each test case.

The remaining experiments are the same as experiment 1 but the targets start from a location $h$ away from the barrier and move along a straight path towards it (in the experiment $h=10 \mathrm{~km}$ ). The difference 


\section{Karatas and Onggo}

between these experiments is in the number of pings that hit the targets while they are on their way to a destination behind the barrier (2, 3, 5, 11 pings, respectively). In a way, a scenario where targets are coming from outside a region that is protected by the barrier provides more opportunities for the sensors to detect the targets because the sensors may ping a few times while the targets are on their way. This creates a more realistic condition. In this scenario, the ABS model has a significant advantage over the INLP model because it is relatively easy to relax the assumptions in the ABS model. It is clear that the results from the INLP model do not provide a good approximation in this more realistic condition. The experiment using the ABS model shows that when the sensors can ping the target multiple times, the performance increases. This increase is significant when the pings hit the targets when they are close to the sensors (the characteristics of a probabilistic detection function). This result shows that when targets are moving, the frequency of sensor pings is an important factor that affects performance. Hence, it should be included in a sensor-barrier model.

\section{CONCLUSION}

In this study we consider the WSN barrier problem and develop an INLP optimization model for generating sensor deployment plans that maximize the total expected detection probability of targets. Different from other studies in the literature, the objective function of our optimization model takes into account issues such as multiple sensor and target types, sensor reliability and probabilistic detection functions. Thus, the analytic model forms a basis for decision-makers to conduct further analysis to measure barrier effectiveness in terms of: (1) sensor quality (reliability and detection probability), (2) trade-offs between sensor quantity and quality, i.e. a large number of unreliable sensors vs a low number of reliable sensors, and (3) sensor-type selection policy (effect of sensor-type selection with respect to the ratio of target types). We believe that using such analytic models in planning WSN barriers can greatly enhance their performance while minimizing sensing holes or weak surveillance zones.

We have shown that the use of ABS for a WSN deployment strategy allows us to explicitly specify the behaviours of sensors and targets and their interactions, which offers an alternative modelling approach. This work has also shown the benefits of complementing an optimization model with an agentbased simulation (ABS) model. The first benefit is that the optimization model can be used to validate the ABS model (and vice versa). Once the ABS model has been validated, it can be used to test the robustness of a solution obtained from the optimization model by relaxing some of the assumptions used in the optimization model to better reflect the reality.

Future work includes the sensitivity analysis of barrier performance (e.g. under varying reliability, sensor numbers, ratios of target types, detection capability, etc.). ABS has a unique characteristic that allows us to explicitly specify individual behaviours and their interactions. Hence, the ABS model can be extended to consider intelligent targets and mobile (and intelligent) sensors. We are also interested to compare the INLP model with some heuristic optimization techniques.

\section{REFERENCES}

Ahmed, N., S. S. Kanhere, and S. Jha. 2005. "Probabilistic coverage in wireless sensor networks." In Conference on Local Computer Networks, pp. 8.

Bereg, S., and D. Kirkpatrick. 2009. "Approximating barrier resilience in wireless sensor networks." Algorithmic Aspects of Wireless Sensor Networks. pp. 29-40. Springer Berlin Heidelberg.

Cardei, M., and Wu, J. 2006. "Energy-efficient coverage problems in wireless ad-hoc sensor networks." Computer communications, 29(4), 413-420.

Chen, A., T. H. Lai, and D. Xuan. 2008. "Measuring and guaranteeing quality of barrier-coverage in wireless sensor networks." In Proceedings of the $9^{\text {th }}$ ACM international symposium on Mobile ad hoc networking and computing. pp. 421-430. 


\section{Karatas and Onggo}

Gage, D. W. 1992. "Command control for many-robot systems." In Proceedings of AUVS-92. Naval Command Control and Ocean Surveillance Center Rdt and E Div San Diego CA. Vol. 10:4, pp. 2834.

He, S., J. Chen, X. Li, X. Shen, and Y. Sun. 2012. "Cost-effective barrier coverage by mobile sensor networks." In Proceedings of INFOCOM, pp. 819-827.

Kumagai, J. 2004. "Life of birds [wireless sensor network for bird study]." Spectrum, 41(4), 42-49.

Kumar, S., T. H. Lai, and A. Arora. 2005. "Barrier coverage with wireless sensors." In Proceedings of the $11^{\text {th }}$ annual international conference on Mobile computing and networking, pp. 284-298.

Kumar, S., T. H. Lai, M. E. Posner, and P. Sinha. 2010. "Maximizing the lifetime of a barrier of wireless sensors." IEEE transactions on mobile computing, (8), 1161-1172.

Kumar, S., T. H. Lai, M. E. Posner, and P. Sinha. 2007. "Optimal sleep-wakeup algorithms for barriers of wireless sensors." IEEE International Conference on Broadband Communications, Networks and Systems, pp. 327-336.

Li, J., J. Chen, and T. H. Lai. 2012. "Energy-efficient intrusion detection with a barrier of probabilistic sensors." In Proceedings of INFOCOM, pp. 118-126.

Liu, B., O. Dousse, J. Wang, and A. Saipulla. 2008. "Strong barrier coverage of wireless sensor networks." In Proceedings of the $9^{\text {th }}$ ACM international symposium on mobile ad hoc networking and computing, pp. 411-420.

Liu, B., and D. Towsley. 2004. "A study of the coverage of large-scale sensor networks." IEEE International Conference on Mobile Ad-hoc and Sensor Systems, pp. 475-483.

Ma, H., M. Yang, D. Li, Y. Hong, and W. Chen. 2012. Minimum camera barrier coverage in wireless camera sensor networks. In Proceedings of INFOCOM. pp. 217-225.

Mustafee, N., and E. E. Bischoff. 2013. Analysing Trade-offs in Container Loading: Combining Load Plan Construction Heuristics with Agent-based Simulation. International Transactions in Operational Research, 20(4), 471-491.

North, M. J., N. T. Collier, J. Ozik, E. R. Tatara, C. M. Macal, M. Bragen, and P. Sydelko. 2013. "Complex adaptive systems modeling with Repast Simphony." Complex Adaptive Systems Modeling, $1(1), 3$.

Saipulla, A., C. Westphal, B. Liu, and J. Wang. 2009. "Barrier coverage of line-based deployed wireless sensor networks." In IEEE INFOCOM, pp. 127-135.

Saipulla, A., B. Liu, and J. Wang. 2008. "Barrier coverage with airdropped wireless sensors." In Military Communications Conference, pp. 1-7.

Ssu, K. F., W. T. Wang, F. K. Wu, and T. T. Wu. 2009. "K-barrier coverage with a directional sensing model." International Journal on Smart Sensing and Intelligent Systems, 2(1), 75-93.

Szewczyk, R., E. Osterweil, J. Polastre, M. Hamilton, A. Mainwaring, and D. Estrin. 2004. "Habitat monitoring with sensor networks." Communications of the ACM, 47(6), 34-40.

$\mathrm{Vu}$, C. T. 2009. "Distributed energy-efficient solutions for area coverage problems in wireless sensor networks." PhD thesis, Georgia State University.

Werner-Allen, G., K. Lorincz, M. Ruiz, O. Marcillo, J. Johnson, J. Lees, and M. Welsh. 2006. "Deploying a wireless sensor network on an active volcano." Internet Computing, 10(2), 18-25.

Yu, L., N. Wang, and X. Meng. 2005. "Real-time forest fire detection with wireless sensor networks." In Proceedings of International Conference on Wireless Communications, Networking and Mobile Computing. Vol. 2, pp. 1214-1217.

Zou, Y., and K. Chakrabarty. 2004. "Sensor deployment and target localization in distributed sensor networks." ACM Transactions on Embedded Computing Systems (TECS) 3, no.1, pp. 61-91.

Zou, Y., and K. Chakrabarty. 2005. A distributed coverage-and connectivity-centric technique for selecting active nodes in wireless sensor networks. IEEE Transactions on Computers, 54(8), 978991. 


\section{AUTHOR BIOGRAPHIES}

MUMTAZ KARATAS graduated from the Turkish Naval Academy in 2001. He received his MS degree in Industrial and Operations Engineering from the University of Michigan and his $\mathrm{PhD}$ in Industrial Engineering from Kocaeli University, Turkey. He spent two years at the Naval Postgraduate School as a visiting researcher and postdoctoral fellow between 2011 and 2013. He is currently an Assistant Professor in the Industrial Engineering Department at the Turkish Naval Academy. His current research areas include optimization and mathematical modelling. His email address is mkaratas@dho.edu.tr.

BHAKTI STEPHAN ONGGO is Lecturer (Assistant Professor) in the Department of Management Science at the Lancaster University Management School (LUMS), Lancaster, United Kingdom. He completed his $\mathrm{PhD}$ in Computer Science at the National University of Singapore and his MSc in Management Science is from Lancaster University. His research interests are in the areas of simulation methodology (conceptual modelling, discrete-event simulation, system dynamics and agent-based simulation) and simulation applications in business and management. His email address is s.onggo@lancaster.ac.uk. 